\title{
Changes in ADMA and TAFI levels after stenting in coronary artery disease patients
}

\author{
NOURAN M. KHALIFA ${ }^{1}$, MOHAMED Z. GAD ${ }^{2}$, ALAA A. HATABA ${ }^{3}$ and LAILA G. MAHRAN ${ }^{1}$ \\ Departments of ${ }^{1}$ Pharmacology and ${ }^{2}$ Biochemistry, Faculty of Pharmacy and Biotechnology, \\ German University in Cairo; ${ }^{3}$ Cardiology Department, Kobry El Kobba Military Hospital, Cairo, Egypt
}

Received February 27, 2012; Accepted June 27, 2012

DOI: $10.3892 / \mathrm{mmr} .2012 .985$

\begin{abstract}
The aim of this study was to examine the contribution of asymmetric dimethylarginine (ADMA), an endogenous inhibitor of nitric oxide synthase (NOS) and a novel marker of vascular endothelial dysfunction, and thrombin-activatable fibrinolysis inhibitor (TAFI), a risk factor for venous thrombosis, to the predisposition of coronary restenosis following stent implantation in coronary artery disease (CAD) patients. Thirty-seven patients with CAD were recruited from the Kobry El Obba Military Hospital, Cairo, Egypt. The patients were hospitalized for coronary angiography and coronary stenting (CS). Overnight fasting blood samples were collected from patients prior to CS and four months later for the determination of plasma ADMA and TAFI levels. The patients underwent follow-up coronary angiography to reveal in-stent restenosis. The results showed that plasma ADMA levels in CAD patients were significantly higher than those reported for healthy subjects. ADMA levels were significantly increased by $30 \%$ in CAD patients four months following CS. CAD patients who developed in-stent restenosis had a $35 \%$ increase in ADMA levels following CS. TAFI levels were not significantly changed after CS in CAD patients or in any of the subgroups. In conclusion, ADMA, but not TAFI, is linked to the predisposition of in-stent restenosis following CS.
\end{abstract}

\section{Introduction}

Percutaneous coronary intervention (PCI), also termed angioplasty, is a safe and effective way to unblock coronary arteries. During this procedure, a catheter is inserted into the groin or arm of the patient and guided forward through the aorta and into the coronary arteries of the heart where stenosed or blocked arteries can be opened with a balloon positioned at

Correspondence to: Professor Mohamed Z. Gad, Department of Biochemistry, Faculty of Pharmacy and Biotechnology, German University in Cairo, New Cairo City 11835, Al Tagamoa Al Khames, Cairo, Egypt

E-mail: mohamed.gad@guc.edu.eg

Key words: asymmetric dimethylarginine, thrombin-activatable fibrinolysis inhibitor, coronary artery disease, stenting and restenosis the tip of the catheter. Initially, angioplasty was performed only with balloon catheters, but technical advances have been made and an improved patient outcome has been achieved with the placement of small metallic spring-like devices called 'stents' at the site of the lesion. The implanted stent serves as a scaffold that keeps the artery open. There are, however, limitations associated with angioplasty and stenting, one of which is known as 'restenosis', which is defined as a recurrence of significant narrowing in the treated vessel (1).

Asymmetric dimethylarginine (ADMA), an endogenous competitive inhibitor of nitric oxide synthase, increases the risk of cardiovascular disease. ADMA inhibits vascular nitric oxide (NO) production at concentrations found in pathophysiological conditions. It also causes local vasoconstriction when infused intra-arterially. ADMA is increased in the plasma of humans with hypercholesterolemia, atherosclerosis, hypertension, chronic renal failure, chronic heart failure and several other disorders. In several prospective and cross-sectional studies, ADMA has evolved as a marker of cardiovascular risk (2). It remains uncertain whether elevations of ADMA levels may play an important pathophysiological role in restenosis that may occur following stent implantation.

The fibrinolytic system is closely correlated with several processes that are involved in restenosis. Thrombin-activatable fibrinolysis inhibitor (TAFI) is a type of fibrinolysis inhibitor that circulates as procarboxypeptidase B2 zymogen, which is converted to active form TAFIa during coagulation. TAFI activation is catalyzed by plasmin, trypsin and the thrombin-thrombomodulin complex. Activated TAFI suppresses fibrinolysis through cleavage of carboxy terminal lysine residues that are expressed during proteolysis of the fibrin polymers, which are the binding sites of plasminogen (3).

The aim of this study was to assess the contribution of ADMA, a novel marker of vascular endothelial dysfunction and atherosclerosis, and TAFI, a risk factor for venous thrombosis, to the predisposition of coronary restenosis following stent implantation in cardiovascular patients.

\section{Materials and methods}

Patients. In total, 37 patients with coronary artery disease (CAD) were recruited from the Department of Cardiology at Kobry El Obba Military Hospital, Cairo, Egypt. The patients were hospitalized for elective coronary angiography and 
PCI, if necessary. Coronary angiography was performed as a consequence of suggested or proven coronary disease by non-invasive techniques. The study protocol was approved by the Local Ethics Committee of Kobry El-Obba Military Hospital in Cairo. Full informed consent was obtained from all patients prior to participation in the study. Exclusion criteria included any concomitant acute or chronic severe diseases such as kidney failure, hepatic insufficiency, acute inflammatory conditions or autoimmune diseases. The routine blood chemistry parameters for risk assessment of ischemic heart disease were performed for each patient on the day of hospitalization.

During PCI, bare metal stents (BMS) and/or drug-eluting stents (DES) were implanted into the coronary vessels depending on the type of culprit coronary lesion, and the co-morbidity of the patients. In total, 15 patients were treated with 20 BMS, and 13 patients were treated with $18 \mathrm{DES}$, and 9 patients were treated with both 13 BMS and 14 DES. ADMA readings of 95 healthy subjects were obtained from the results of a previous study conducted in our laboratory (4).

Sampling and storage. Prior to PCI or elective coronary angiography, overnight fasting blood samples were collected from the patients in order to determine ADMA and TAFI plasma levels. All the patients were undergoing Plavix (clopidogrel) treatment and samples were obtained prior to anticoagulant (heparin) injection. Samples were collected in vacutainer tubes containing $\mathrm{Na}_{2}$ EDTA. Plasma samples were separated by centrifugation at $3000 \mathrm{rpm}$ for $15 \mathrm{~min}$. Aliquots of collected plasma were stored at $-70^{\circ} \mathrm{C}$ until analysis of ADMA and TAFI was undertaken.

Prior to PCI, combined antithrombotic treatment was administered to each patient, i.e., 300-600 mg clopidogrel and $60 \mathrm{IU} / \mathrm{kg}$ sodium-heparin. Invasive examinations (coronary and angiography) were performed using a Philips BH 5000 monoplane or biplane system. Recordings were subsequently analyzed by two well-trained invasive cardiologists using Philips QCA software.

Semi-qualitative analyses were performed independently by two experienced interventional cardiologists. The location of the restenotic lesions with respect to the stented segment was classified as being within the stent body or at the proximal or distal margin of the stent. Stenosis within the stent or at the margins was further classified as being either diffuse or focal.

As part of a routine follow-up program to detect restenosis 4 months following successful CS, patients underwent followup diagnostic coronary angiography. Fasting blood samples were collected from the patients on EDTA prior to the angiographic follow up and centrifuged at $3000 \mathrm{rpm}$ for $20 \mathrm{~min}$. The plasma aliquots were then stored at $-70^{\circ} \mathrm{C}$ until analysis of ADMA and TAFI.

Determination of ADMA and TAFI in plasma samples. Quantitative determination of ADMA and TAFI in plasma was performed by the ELISA technique using an ADMA $^{\circledR}$ - ELISA kit supplied by DLD Diagnostika GmbH (Hamburg, Germany) and IMUBIND ${ }^{\circledR}$ TAFIa/ai Antigen ELISA kit supplied by American Diagnostica Inc. (ADI) (Stamford, CT, USA).

Statistical analysis. Statistical analysis was performed using the statistical package for social sciences (SPSS, Chicago, IL,
Table I. Clinical, angiographic and procedural characteristics of the studied groups.

Characteristics

Age (years), mean \pm SEM

$55.3 \pm 1.45$

Total no. of patients, $\mathrm{n}$

Patients with angiographic

29 (78.4)

follow up, n (\%)

Patients with multislice

CT scan follow up, n (\%)

Diabetic patients, n (\%)

Non-diabetic patients, n (\%)

Hypertensive, n (\%)

Non-hypertensive, n (\%)

Patients with bare

metal stent (BMS), $\mathrm{n}$

Patients with drug-eluting

stent (DES), $\mathrm{n}$

Smokers, $\mathrm{n}$

Current smokers 8

Quit around 5 months before PCI

Non-smokers, $\mathrm{n}$

Patients with patent stents, $n$

Patients with in-stent restenosis

( $<50 \%$ of artery diameter), $n$

Patients with in-stent restenosis

( $>50 \%$ of artery diameter), $\mathrm{n}$

13

PCI, percutaneous coronary intervention.

USA) version 19 and GraphPad Prism version 5.5. Data were presented as the mean values \pm SEM. $\mathrm{P}<0.05$ was considered to indicate a statistically significant difference. Categorical variables were analyzed using the Pearson's Chi-square test, whereas continuous variables were evaluated using the Student's t-test to compare coronary risk parameters of the stent group prior to CS and during follow-up coronary angiography. One-way ANOVA tests were performed for comparison among the different groups.

\section{Results}

Patient characteristics. Table I shows the clinical, angiographic and procedural characteristics of the studied patients. It also shows that $37.8 \%$ of patients had no in-stent restenosis four months following stent placement. However, the remaining patients $(62.2 \%)$ were subjected to in-stent restenosis either with $<50 \%$ of artery diameter $(27.0 \%$ of patients) or $>50 \%$ (35.2\% of patients).

Coronary stenting and plasma ADMA levels. Results showed that the mean plasma ADMA level was significantly higher by $19.73 \%$ in CAD patients ( $n=37)$ as compared with the healthy control subjects $(n=95 ; P=0.0021)$ (Fig. 1). In addition, in CAD 


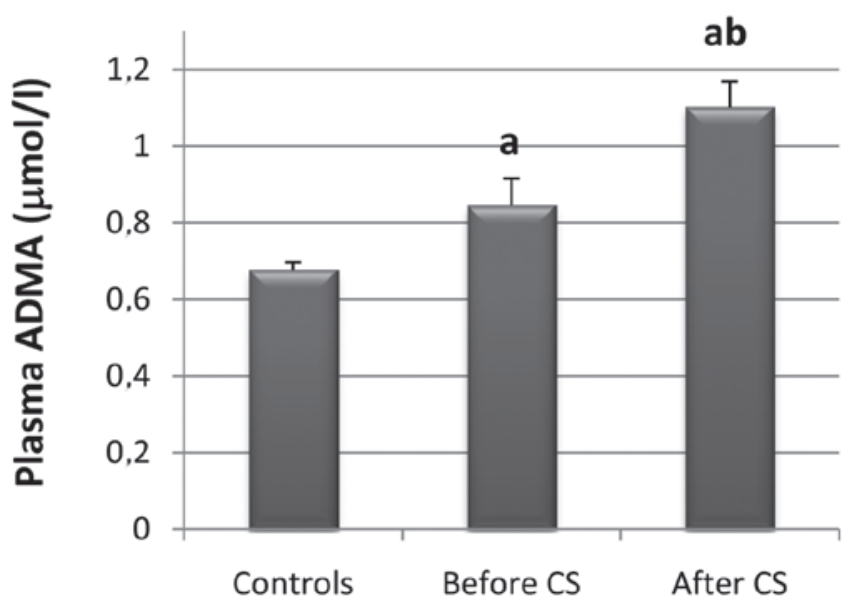

Figure 1. Plasma ADMA levels in healthy $(\mathrm{n}=95)$ and CAD patients prior to and after coronary stenting (CS) $(n=37)$. Data are the means \pm SEM. ${ }^{a}$ Sig nificantly different from mean ADMA level in control; $\mathrm{p}<0.05$. ${ }^{\mathrm{b}}$ Significantly different from mean ADMA level prior to CS; $\mathrm{p}<0.05$. ADMA, asymmetric dimethylarginine; $\mathrm{CAD}$, coronary artery disease.

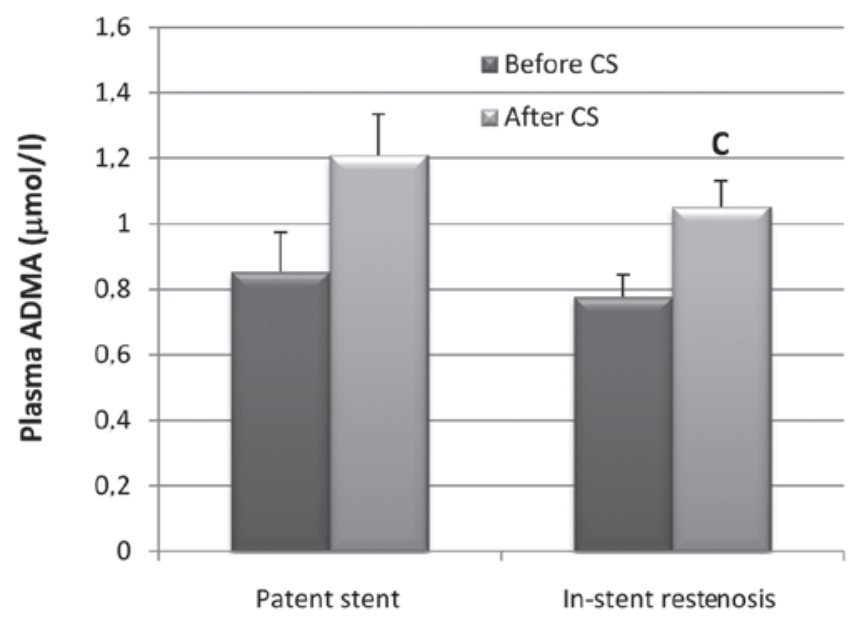

Figure 2. Plasma levels of ADMA prior to and following CS in CAD patients with patent stents $(n=14)$ and in-stent restenosis $(n=23)$. Data are the means \pm SEM. ${ }^{c}$ Significantly different from mean ADMA level before CS in CAD with in-stent restenosis; $\mathrm{p}<0.05$. ADMA, asymmetric dimethylarginine; $\mathrm{CS}$, coronary stenting; $\mathrm{CAD}$, coronary artery disease.

patients the ADMA levels were significantly higher by $23.33 \%$ following $\mathrm{CS}$ as compared to those prior to $\mathrm{CS}(\mathrm{P}=0.0123)$.

In-stent restenosis and plasma ADMA levels. In the $14 \mathrm{CAD}$ patients with patent stents, ADMA levels prior to CS were not significantly different from those levels after CS $(\mathrm{P}=0.0535)$. However, in all CAD patients with in-stent restenosis $(n=23)$, ADMA levels after CS were found to be significantly higher by $26.16 \%$ than those levels before $\mathrm{CS}(\mathrm{P}=0.0102)$ (Fig. 2).

It was also revealed that plasma ADMA levels prior to CS in patients with $>50 \%$ in-stent restenosis were significantly higher by $32.3 \%$ than ADMA levels prior to CS in patients with $<50 \%$ in-stent restenosis $(\mathrm{P}=0.0327$ ) (Fig. 3).

Coronary stenting and plasma TAFI levels. It is of note that in all 37 CAD patients, whether with patent stent or in-stent

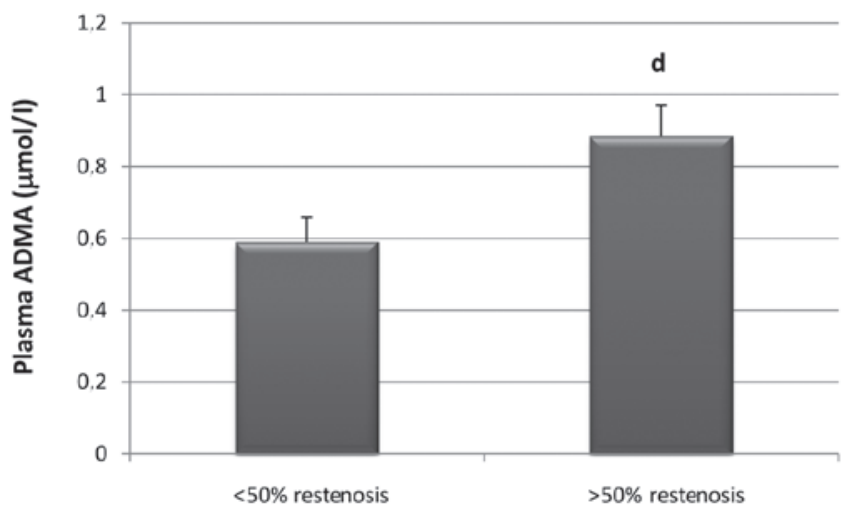

Figure 3. Plasma ADMA levels in patients with less than $(n=10)$ and more than $(n=13) 50 \%$ in-stent restenosis. Data are the means \pm SEM. ${ }^{\text {SSignifi- }}$ cantly different from the mean ADMA level prior to CS in CAD with in-stent restenosis; $\mathrm{p}<0.05$. ADMA, asymmetric dimethylarginine; CS, coronary stenting; CAD, coronary artery disease.

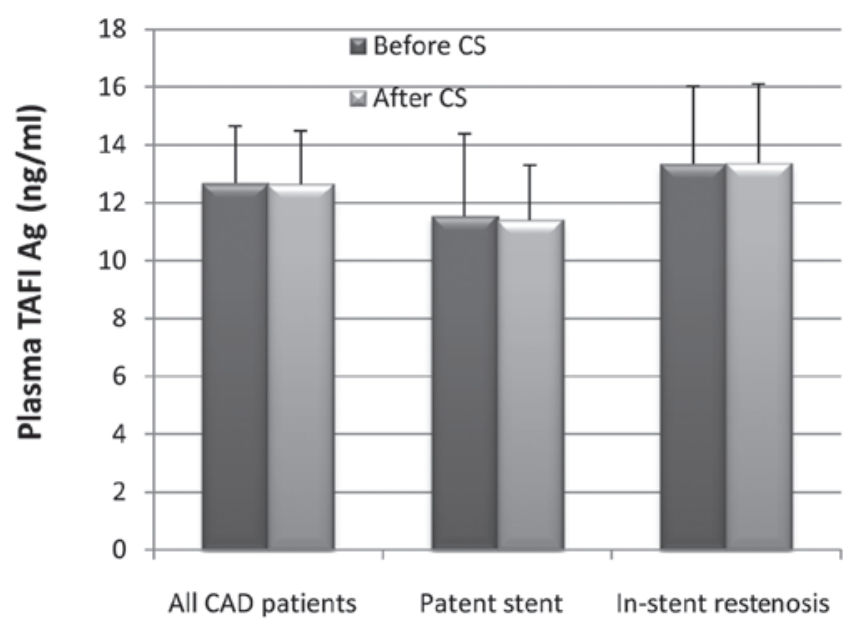

Figure 4. Plasma TAFI levels in all CAD patients ( $n=37)$, CAD patients with patent stent $(n=14)$, and CAD patients with in-stent restenosis $(n=23)$ prior to and following coronary stenting (CS). TAFI, thrombin-activatable fibrinolysis inhibitor; CAD, coronary artery disease.

restenosis, or pre- or post-CS, the plasma TAFI levels did not significantly change (Fig. 4).

\section{Discussion}

NO secreted by the endothelium is important in ensuring cardiac homeostasis. NO provides basal tonus by relaxing vascular smooth muscle cells (5). It inhibits the adhesion of leukocytes and thrombocytes, as well as the activation and aggregation of the latter. Additionally, NO inhibits the formation of superoxide radicals, oxidation of low-density lipoproteins, and the migration and proliferation of smooth muscle cells (6-8). Decreased levels of NO initiate atherosclerosis and lead to its exacerbation $(9,10)$. Decreased NO activity, also thought to be one of the factors responsible for restenosis, develops following arterial injury (11). Restenosis following balloon angioplasty is a consequence of neointimal hyperplasia and vessel remodeling. NO inhibits neointimal hyperplasia. 
ADMA is a substance that inhibits NO synthesis by blocking the NOS enzyme. High concentrations of plasma ADMA may be used to predict the severity of coronary atherosclerosis and the development of CAD (12). Increased ADMA concentration produces elevation in aortic and arterial blood pressure. Elevated blood pressure is associated with the presence of coronary artery narrowing (13). A multicentered, case-control CARDIAC (Coronary Artery Risk Determination investigating the effect of ADMA Concentration) study revealed that every $1 \mu \mathrm{mol} / 1$ increase in plasma ADMA concentration led to a 2.35-fold increase in the risk of CAD (14). Our study supports previous reports and demonstrates the significant elevation of mean plasma ADMA levels $(\mathrm{P}=0.0021)$ in $\mathrm{CAD}$ patients as compared to control healthy subjects. The observation that ADMA levels increase early in the development of atherosclerosis suggests that ADMA has the potential to be both a marker and a mediator of vascular lesions (15).

Sahinarslan et al (16) investigated the correlation of plasma ADMA concentration with lesion distribution and severity at coronary artery angiography. Those authors enrolled patients with stable angina and divided them into two groups. Group I included the patients with normal coronary arteries. All other patients were included in group II. Results of that study showed that ADMA levels were higher in group II compared to group I, and the L-arginine/ADMA ratio was lower in group II patients compared to group I patients. ADMA positively correlated with the coronary atherosclerotic score. Moreover, ADMA was an important predictor of angiographically defined CAD. Thus, those authors (16) supported the hypothesis that plasma ADMA concentration is a good predictor of CAD.

Our results showed that ADMA levels were significantly higher $(\mathrm{P}=0.0123)$ by $23.33 \%$ in CAD patients $(\mathrm{n}=37)$ four months after CS as compared to levels prior to stenting. This finding may be attributed to the increased ADMA levels after myocardial ischemia-reperfusion injury (17). This study also revealed a significant increase of $26.16 \%$ in plasma ADMA levels following CS in all CAD patients with in-stent restenosis. This observation was not valid for patients with patent stent, which may prove a positive correlation between increased ADMA levels and the incidence of cardiovascular events and in-stent restenosis. In contrast to our findings, Ajtay et al (18) demonstrated that in patients with CAD, PCI stent placement markedly decreased the plasma level of ADMA. Coronary angiography alone resulted in an increase of ADMA.

Krempl et al (19) found that ADMA is significantly elevated in patients with unstable angina. A reduced ADMA level at 6 weeks after PCI may indicate a decreased risk of recurrent cardiovascular events. In their study, Krempl et al (19) investigated the role of ADMA in the clinical outcome of patients with unstable angina. Their results showed that baseline ADMA concentration in controls was significantly lower than that in patients with CAD. Additionally, patients with unstable angina had significantly higher baseline ADMA levels than patients with stable angina and there was a significant reduction in ADMA levels at 6 weeks after PCI in patients with unstable angina who experienced no recurrent cardiovascular event. By contrast, those authors found that in patients who experienced another acute event following CS, ADMA concentrations remained elevated 6 weeks after PCI.
Results of multivariate analysis indicated that plasma ADMA independently almost tripled the risk for recurrent symptomatic stenosis of an arteriovenous fistula (AVF) following percutaneous transluminal angioplasty. These results suggest the involvement of ADMA in the progression of symptomatic restenosis of AVFs after PCI and require preventive strategies that target ADMA and/or endothelial dysfunction to decrease the risk for AVF restenosis (20).

TAFI is a recently described inhibitor of fibrinolysis which is involved in the regulation of the balance between coagulation and fibrinolysis. High TAFI plasma levels may therefore contribute to a hypo-fibrinolytic state and to an increased risk of thrombotic disorders. There are contradictory results regarding TAFI levels in CAD patients, possibly due to the differences in the characteristics of patients and the time of blood sampling among different studies (21).

The fibrinolytic system is closely correlated with several processes that are involved in restenosis. In the study by Lau et al (3), it was found that high pre-procedural plasma levels of TAFI antigen increased the risk of restenosis following PCI. Animal studies have shown that inhibition of the early initiators of the coagulation pathway, such as factor VII and tissue factor, decrease late neointimal hyperplasia (22). Therefore, a potential target for the inhibition of restenosis is to limit early thrombosis. High levels of TAFI have been associated with deep venous thrombosis (23) and CAD (24). Increased TAFI levels were found to be a risk factor for the development of angina pectoris among apparently healthy males (25). A high TAFI level was associated with a 2-fold increase in the risk of recurrence of venous thrombosis in comparison with patients with lower TAFI levels (26). Contradictory results have been reported for the role of TAFI in myocardial infarction. Previous studies have shown that patients with a recent myocardial infarction presented lower values of TAFI antigen while elevated TAFI levels were actually protective against myocardial infarction $(27,28)$.

As the list of substrates for TAFI grows, it becomes clear that besides the regulation of fibrinolysis, TAFI is likely to play a potentially important role in processes such as blood pressure regulation, inflammation and wound healing. Different studies markedly suggest that besides being important in the regulation of fibrinolysis, TAFI may also have an important function in the regulation of inflammation. High TAFI levels are protective against arterial thrombosis by inactivation of inflammatory mediators such as bradykinin and C5a (29).

Paola Cellai et al (21) measured plasma TAFI activity and antigen levels in 44 patients admitted to the Coronary Care Unit and in a group of 44 healthy controls, matched for age and gender, to detect a possible association of their levels with acute CAD. No differences were found in TAFI, either at the activity or antigen levels, between patients and controls.

Our data revealed that the difference between TAFI levels prior to and following CS in all CAD patients was not significant. Moreover, our findings have shown that TAFI levels prior to CS in CAD patients with in-stent restenosis were not significantly different from those after CS, indicating that TAFI does not play a role in in-stent restenosis.

In conclusion, the salient findings of our study are as follows: i) The mean plasma level of ADMA is significantly higher in CAD patients than that reported for healthy subjects, 
ii) plasma ADMA levels are significantly higher in CAD patients four months after CS as compared to levels prior to stenting, iii) CAD patients who developed in-stent restenosis during angiographic follow-up had a significant increase in ADMA levels following CS, in contrast to patients with patent stent who did not show this significant increase, iv) plasma ADMA levels prior to stenting in patients with $>50 \%$ in-stent restenosis were significantly higher than those in patients who developed $<50 \%$ in-stent restenosis; v) in non-diabetic CAD patients who developed in-stent restenosis, ADMA levels were higher by $19 \%$ after CS and vi) TAFI levels did not significantly change following CS in CAD patients. Therefore, we can conclude that ADMA, but not TAFI, is correlated with the pre-disposition of in-stent restenosis following CS.

\section{References}

1. Dangas G and Kuepper F: Cardiology patient page. Restenosis: repeat narrowing of a coronary artery: prevention and treatment. Circulation 105: 2586-2587, 2002.

2. Boger RH: Asymmetric dimethylarginine (ADMA) and cardiovascular disease: insights from prospective clinical trials. Vasc Med 10: S19-S25, 2005.

3. Lau HK, Segev A, Hegele RA, Sparkes JD, Teitel JM, et al: Thrombin-activatable fibrinolysis inhibitor (TAFI): a nove predictor of angiographic coronary restenosis. Thromb Haemost 90: 1187-1191, 2003.

4. Gad MZ, Hassanein SI, Abdel-Maksoud SM, Shaban GM, Abou-Aisha K, et al: Assessment of serum levels of asymmetric dimethylarginine (ADMA), symmetric dimethylarginine (SDMA) and L-arginine in coronary artery disease. Biomarkers 15: 746-752, 2010.

5. Loscalzo J and Welch G: Nitric oxide and its role in the cardiovascular system. Prog Cardiovasc Dis 38: 87-104, 1995.

6. Hogg N, Kalyanaraman B, Joseph J, Struck A and Parthasarathy S: Inhibition of low-density lipoprotein oxidation by nitric oxide. Potential role in atherogenesis. FEBS Lett 334: 170-174, 1993.

7. Marks DS, Vita JA, Folts JD, Keaney Jr JF, Welch GN, et al Inhibition of neointimal proliferation in rabbits after vascular injury by a single treatment with a protein adduct of nitric oxide. J Clin Invest 96: 2630-2638, 1995.

8. Garg UC and Hassid A: Nitric oxide-generating vasodilators and 8-bromo-cyclic guanosine monophosphate inhibit mitogenesis and proliferation of cultured rat vascular smooth muscle cells. J Clin Invest 83: 1774-1777, 1989.

9. Valkonen VP, Paiva H, Salonen JT, Lakka TA, Lehtimaki T, et al: Risk of acute coronary events and serum concentration of asymmetrical dimethylarginine. Lancet 358: 2127-2128, 2001.

10. Zoccali C, Bode-Boger S, Mallamaci F, Benedetto F, Tripepi G, et al: Plasma concentration of asymmetrical dimethylarginine and mortality in patients with end-stage renal disease: a prospective study. Lancet 358: 2113-2117, 2001.

11. Janero DR and Ewing JF: Nitric oxide and postangioplasty restenosis: pathological correlates and therapeutic potential. Free Radic Biol Med 29: 1199-1221, 2000.

12. Lu TM, Ding YA, Charng MJ and Lin SJ: Asymmetrical dimethylarginine: a novel risk factor for coronary artery disease. Clin Cardiol 26: 458-464, 2003.

13. Wykretowicz A,MetzlerL,Milewska A,Balinski M,Rutkowska A, et al: Noninvasively assessed pulsatility of ascending aortic pressure waveform is associated with the presence of coronary artery narrowing. Heart Vessels 23: 16-19, 2008.
14. Schulze F, Lenzen H, Hanefeld C, Bartling A, Osterziel KJ, et al: Asymmetric dimethylarginine is an independent risk factor for coronary heart disease: results from the multicenter Coronary Artery Risk Determination investigating the Influence of ADMA Concentration (CARDIAC) study. Am Heart J 152: 493 e1-8, 2006.

15. Boger RH: Asymmetric dimethylarginine, an endogenous inhibitor of nitric oxide synthase, explains the 'L-arginine paradox' and acts as a novel cardiovascular risk factor. J Nutr 134: 2842S-2847S, 2004.

16. Sahinarslan A, Cengel A, Biberoglu G, Hasanoglu A, Turkoglu S, et al: Plasma asymmetric dimethylarginine level and extent of lesion at coronary angiography. Coron Artery Dis 17: 605-609, 2006.

17. Cziraki A, Ajtay Z, Nemeth A, Lenkey Z, Sulyok E, et al: Effects of coronary revascularization with or without cardiopulmonary bypass on plasma levels of asymmetric dimethylarginine. Coron Artery Dis 22: 245-252, 2011.

18. Ajtay Z, Scalera F, Cziraki A, Horvath I, Papp L, et al: Stent placement in patients with coronary heart disease decreases plasma levels of the endogenous nitric oxide synthase inhibitor ADMA. Int J Mol Med 23: 651-657, 2009.

19. Krempl TK, Maas R, Sydow K, Meinertz T, Boger RH, et al: Elevation of asymmetric dimethylarginine in patients with unstable angina and recurrent cardiovascular events. Eur Heart J 26: 1846-1851, 2005.

20. Wu CC, Wen SC, Yang CW, Pu SY, Tsai KC, et al: Plasma ADMA predicts restenosis of arteriovenous fistula. J Am Soc Nephrol 20: 213-222, 2009.

21. Paola Cellai A, Antonucci E, Alessandrello Liotta A, Fedi S, Marcucci R, et al: TAFI activity and antigen plasma levels are not increased in acute coronary artery disease patients admitted to a coronary care unit. Thromb Res 118: 495-500, 2006.

22. Jang Y, Guzman LA, Lincoff AM, Gottsauner-Wolf M, Forudi F, et al: Influence of blockade at specific levels of the coagulation cascade on restenosis in a rabbit atherosclerotic femoral artery injury model. Circulation 92: 3041-3050, 1995.

23. van Tilburg NH, Rosendaal FR and Bertina RM: Thrombin activatable fibrinolysis inhibitor and the risk for deep vein thrombosis. Blood 95: 2855-2859, 2000.

24. Silveira A, Schatteman K, Goossens F, Moor E, Scharpe S, et al: Plasma procarboxypeptidase $\mathrm{U}$ in men with symptomatic coronary artery disease. Thromb Headmost 84: 364-368, 2000.

25. Morange PE, Juhan-Vague I, Scarabin PY, Alessi MC, Luc G, et al: Association between TAFI antigen and Ala147Thr polymorphism of the TAFI gene and the angina pectoris incidence. The PRIME Study (Prospective Epidemiological Study of MI). Thromb Haemost 89: 554-560, 2003.

26. Eichinger S, Schonauer V, Weltermann A, Minar E, Bialonczyk C, et al: Thrombin-activatable fibrinolysis inhibitor and the risk for recurrent venous thromboembolism. Blood 103: 3773-3776, 2004.

27. Juhan-Vague I, Morange PE, Aubert H, Henry M, Aillaud MF, et al: Plasma thrombin-activatable fibrinolysis inhibitor antigen concentration and genotype in relation to myocardial infarction in the north and south of Europe. Arterioscler Thromb Vasc Biol 22: 867-873, 2002.

28. Juhan-Vague I and Morange PE: Very high TAFI antigen levels are associated with a lower risk of hard coronary events: the PRIME Study. J Thromb Haemost 1: 2243-2244, 2003.

29. Bouma BN and Mosnier LO: Thrombin activatable fibrinolysis inhibitor (TAFI) at the interface between coagulation and fibrinolysis. Pathophysiol Haemost Thromb 33: 375-381, 2003. 